\title{
Dark Current Compensation and Sensitivity Adjustment on Gallium Arsenide Linear Array Detector for X-Ray Imaging
}

\section{Mikhail Polkovnikov}

Department of Experimental Physics, Institute for High Energy Physics (IHEP), National Research Center "Kurchatov Institute", Protvino, Russia

Email:pmk@ihep.ru

How to cite this paper: Polkovnikov, M. (2016) Dark Current Compensation and Sensitivity Adjustment on Gallium Arsenide Linear Array Detector for X-Ray Imaging. J. Biomedical Science and Engineering, 9, 532-543.

http://dx.doi.org/10.4236/jbise.2016.911047

Received: July 29, 2015

Accepted: October 28, 2016

Published: October 31, 2016

Copyright () 2016 by author and Scientific Research Publishing Inc. This work is licensed under the Creative Commons Attribution International License (CC BY 4.0).

http://creativecommons.org/licenses/by/4.0/

\section{Abstract}

For the last several years, the linear array $\mathrm{x}$-ray detector for $\mathrm{x}$-ray imaging with gallium arsenide direct conversion sensitive elements has been developed and tested at the Institute for High Energy Physics. The array consists of 16 sensitive modules. Each module has 128 gallium arsenide (GaAs) sensitive elements with $200 \mu \mathrm{m}$ pitch. Current article describes two key program procedures of initial dark current compensation of each sensitive element in the linear array, and sensitivity adjustment for alignment of strip pattern in the raw image data. As a part of evaluation process a modular transfer function (MTF) was measured with the slanted sharp-edge object under RQA5 technique as it described in the International Electrotechnical Commission 62220-1 standard (high voltage $70 \mathrm{kVp}$, additional aluminium filter $21 \mathrm{~mm}$ ) for images with compensated dark currents and adjusted sensitivity of detector elements. The 10\% level of the calculated MTF function has spatial resolution within 2 - 3 pair of lines per $\mathrm{mm}$ for both vertical and horizontal orientation.

\section{Keywords}

Linear Array, Gallium Arsenide, Calibration, Dark Current, Sensitivity, Modular Transfer Function, Normalized Noise Power Spectrum

\section{Introduction}

The using of semiconductor materials with direct conversion of $\mathrm{x}$-ray photon energy into electron charges is the one of the primary techniques in developing modern detector systems for different kinds of applications. System based on selenium and cadmium telluride (CdTe and CZT) semiconductors are being used for medical purpose and de- 
structive testing both in current integration and counting mode. In other hand the gallium arsenide ( $\mathrm{GaAs}$ ) material doesn't used in commercial $\mathrm{x}$-ray detection systems yet, but there were several examples of linear arrays [1]-[4] developed in the past decade. All of them were using different types of detector sensitive elements arrangement into array, different ASIC to form raw data for acquisition system and different acquisition system elements to form an actual image. The formation of the images in those systems requires several procedures to transform raw data into image "for processing" as DICOM 3.0 standard requires for medical imaging. In case of linear array detectors with a direct conversion there are two sources of non-uniformity: the raw sequence of signals formed by different dark current of each element without $\mathrm{x}$-ray flux and sensitivity of each element to $x$-ray exposure. There are many different algorithms to reduce this type non-uniformity which available mainly for infrared imaging systems [5]-[8] and because infrared linear arrays have very similar non-uniformity pattern. But in case of $\mathrm{x}$-ray imaging such algorithms are the proprietary methods, unavailable as software libraries. This article will focus on two algorithms of software dark current and of detector elements sensitivity compensation developed for the GaAs arrays.

\section{Detector Array Structure and Adjustment}

\subsection{Linear Array Structure}

The key unit of the linear array is a module, consisting of 128 sensitive elements of gallium arsenide chromium doped resistive type material which was designed, manufactured and assembled in Siberian Physical-Technical Institute of Tomsk State University [9] [10]. The pitch size of a single element can be $100 \times 100 \mu \mathrm{m}^{2}$ or $200 \times 200 \mu \mathrm{m}^{2}$ and the sensitive length of material vary from $600 \mu \mathrm{m}$ for pad elements up to $4 \mathrm{~mm}$ for strip elements. Figure 1 shows two detectors modules of strip sensitive elements with 200 $\mu \mathrm{m}$ pixel pitch, mounted on the printed circuit board. Arrows show the direction of photon flux. The maximum array length was $400 \mathrm{~mm}$ assembled from 16 modules. Due to technological reasons is it impossible to make all the modules with same sensitivity
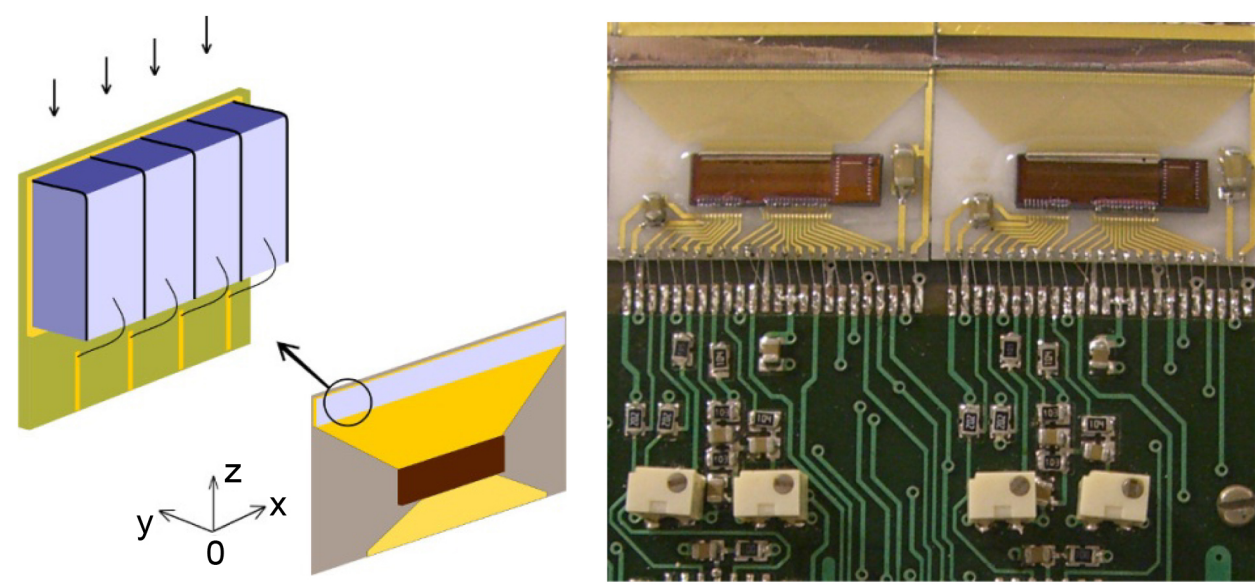

Figure 1. Simplified example of strip sensitive elements arrangement on detector module and two detector modules on an acquisition board. 
to the $\mathrm{x}$-ray exposure, moreover the sensitivity of pads and strips vary not only from one module to another but also inside each module. The transformation of signals from detectors into useful voltage signal is done by 128-channel ASIC chip [11]. Every channel of ASIC consists of: input circuit for matching of bias voltage of sensitive element, personal 8-bit compensation current generator and 8-bit storage memory, compensation current generator for all channels, integrator and buffer. ASIC output doesn't have any programmable gain amplifiers to adjust the sensitivity of each element. That is the reason why we have to do a programmable software adjustment. The connection between sensitive elements and ASIC is made by ultrasound welding. The chip is converting the electron charges, formed by the $\mathrm{x}$-ray flux into serial sequence of voltages.

\subsection{Principle of Control and Adjustment}

There are two possibilities to control the output serial voltage signals of ASIC. The first one is to adjust the average level of output signals and the second one is to adjust input current for every sensitive element to minimize the spread of dark currents of all detectors on the module. To adjust both average level of output signals value and individual dark currents the chip has several analog and digital inputs. The adjustment of the mean level is controlled manually with the potentiometer and with the software regulations of the dark currents by writing 8-bit value codes in ASIC. These two possibilities allow control a shadow signals only when there is no $\mathrm{x}$-ray flux.

For each sensitive element there is a transfer characteristic function of output voltage in dependence of input 8-bit code from 0 to 255. By calculation of optimal input codes (sometimes it's called a mask) it is possible to get output serial voltage signals as a straight line. The current of each input of ASIC includes three components, they are: $I_{d e t}$-current formed by sensitive element under x-ray exposure, $I_{1}$-average current of all 128 sensitive elements, $I_{2}$-compensation current, $k$-input 8 -bit value. The output voltage signal for every one is proportional to input current and clock frequency.

$$
\begin{gathered}
I_{\text {input }}=I_{\text {det }}+I_{1}-k \cdot I_{2} \\
U_{\text {output }} \propto \frac{K \cdot I_{\text {input }}}{f_{c l k}}
\end{gathered}
$$

Clock frequency is constant during the exposure and acquisition. Voltage sequence transformed by ADC into counts has a form as it showed in a Figure 2.

The ADC count has three main ranges (3), two of them are constants, and one is the working range where the dark current can be compensated.

$$
A_{\text {out }}= \begin{cases}A_{\max }, & N \in\left[0, N_{0}\right] \\ A_{\max }-N \cdot \operatorname{tg}(\alpha), & N \in\left(N_{0}, N_{\max }\right) \\ 0, & N \in\left[N_{\max }, 255\right]\end{cases}
$$

To make all signals have the same or close to same $A_{\text {base }}$ count value it's necessary to calculate $N_{\text {base }}$ input value for each chip sensitive element input. To do that it's necessary to form a response matrix $A$. 


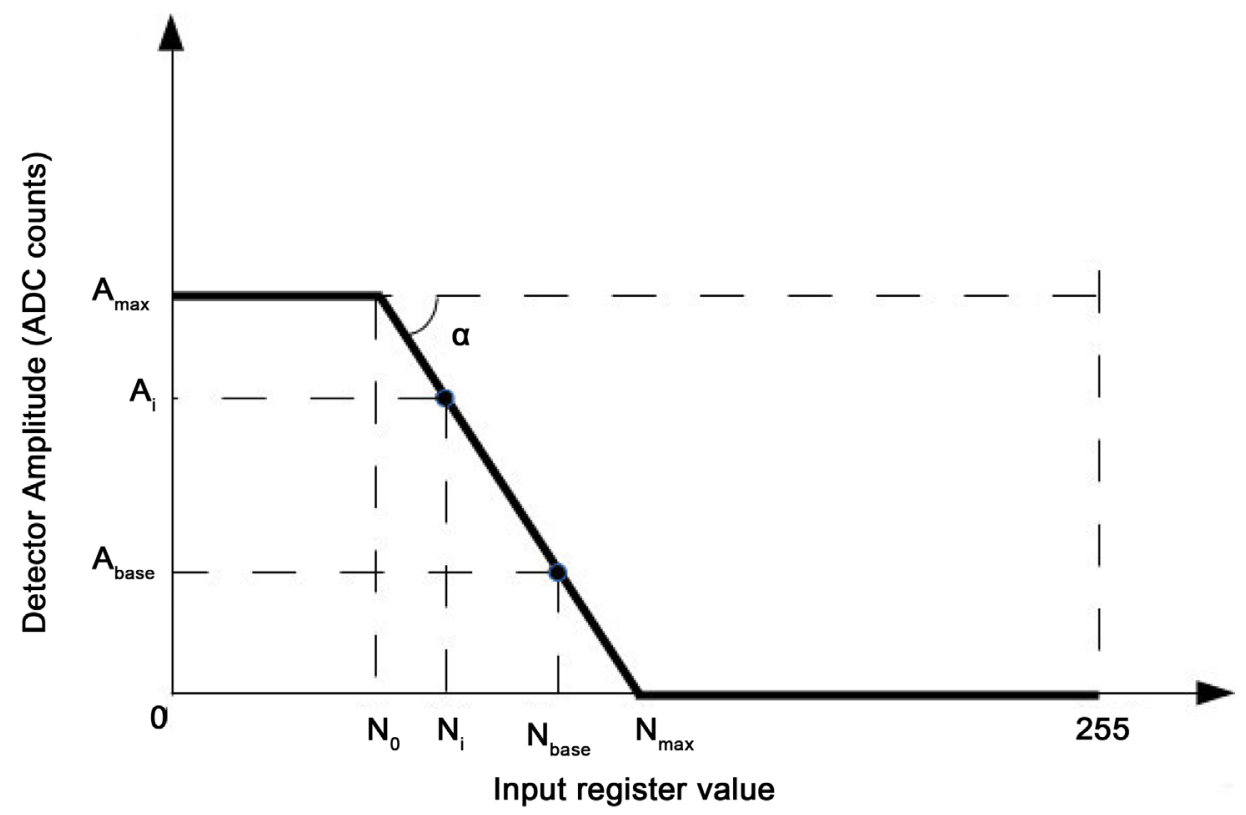

Figure 2. Transfer characteristic of the ADC count of the single sensitive element according to 8-digit input code of ASIC.

$$
A_{m, n}=\left(\begin{array}{cccc}
a_{0,1} & a_{1,2} & \cdots & a_{1, n} \\
a_{1,1} & a_{2,2} & \cdots & a_{2, n} \\
\vdots & \vdots & \ddots & \vdots \\
a_{m, 1} & a_{m, 2} & \cdots & a_{m, n}
\end{array}\right)
$$

where $m$ is number of input values in chip, and $n$ is amount of sensitive elements. In our case the maximum $m$ is equal to 255 and $n$ is equal to 128 . The $m$ values could be an array of equidistant distribution with different step from 0 to 255 , e.g.

$m=(0,5,10, \cdots, 255)$.

The easiest way to find the $N_{j}=N_{\text {base }}$ or it nearest neighbor is to calculate the index of minimum absolute difference between $A_{\text {base }}$ and each element of column vector $A$.

$$
\begin{aligned}
& \forall j \in\{1,2, \cdots, n\}: \bar{A}_{j}=\left(\begin{array}{c}
\left|a_{0, j}-A_{\text {base }}\right| \\
\left|a_{1, j}-A_{\text {base }}\right| \\
\vdots \\
\left|a_{m, j}-A_{\text {base }}\right|
\end{array} \mid\right. \\
& \forall i \in\{0,1, \cdots, m\} \exists N_{j}: N_{j}=\arg \min _{i}\left(\bar{A}_{j}\right)
\end{aligned}
$$

where

$$
\begin{aligned}
& \arg \min _{x} f(x)=\left\{x \mid f(x)=\min _{x^{\prime}} f\left(x^{\prime}\right)\right\} \\
& \min _{x} f(x)=\left\{f(x) \mid f(x)<f\left(x_{0}\right) \forall x_{0} \in R\right\}
\end{aligned}
$$

After finding all compensation values for the array and writing them into ASICs, in result the output voltage signal will be with minimum spread. If an equidistant distri- 
bution step not equal to 1 the Figure 3 shows the spread of ADC counts from a single module.

\subsection{Compensation of Different Sensitive Elements X-Ray Response}

However, there is still a different sensitivity of detector elements to $\mathrm{x}$-ray flux, even when all values for dark current compensation are correct and output signals without $\mathrm{x}$-ray flux are a uniform. That pattern depends on many parameters such as bias voltage of detector elements, sampling clock frequency, high voltage of $\mathrm{x}$-ray tube etc. To reduce such pattern we use a calibration test-object which has a shape of a ramp. The idea of sensitivity adjustment is based on a calculated response from a reference uniform signal for different intensities. Such shape allows to simulate the variation of $\mathrm{x}$-ray flux intensity and get a response of each sensitive element under the same uniform $\mathrm{x}$-ray flux. In our case the maximum difference in the ramp thickness is $20 \mathrm{~mm}$ that covers the major part of studies where $\mathrm{x}$-ray high voltage is equal or less $70 \mathrm{kVp}$. Figure 4 demonstrates the ramp object scanned from top to bottom by the linear array with 5 detector modules. The data presented after dark current compensation. The vertical pattern represents the different sensitivity from one detector element to another.

For a typical $\mathrm{x}$-ray tube that has a bremsstrahlung spectrum as well as characteristic lines, the mono-energetic formula of intensity attenuation like (6)

$$
I=I_{0} \mathrm{e}^{-\mu \cdot x}
$$

can't be used to calculate the response of each sensitive element and compensate it, thus the response of each sensitive element is represented as a polynomial dependency (7), where $d$ is a ramp thickness and $k_{i}$ are coefficients, which allows to take into account both the scattering and absorption of $\mathrm{x}$-ray photons.

$$
I=\sum_{i=0}^{3} k_{i} \cdot d^{i}
$$

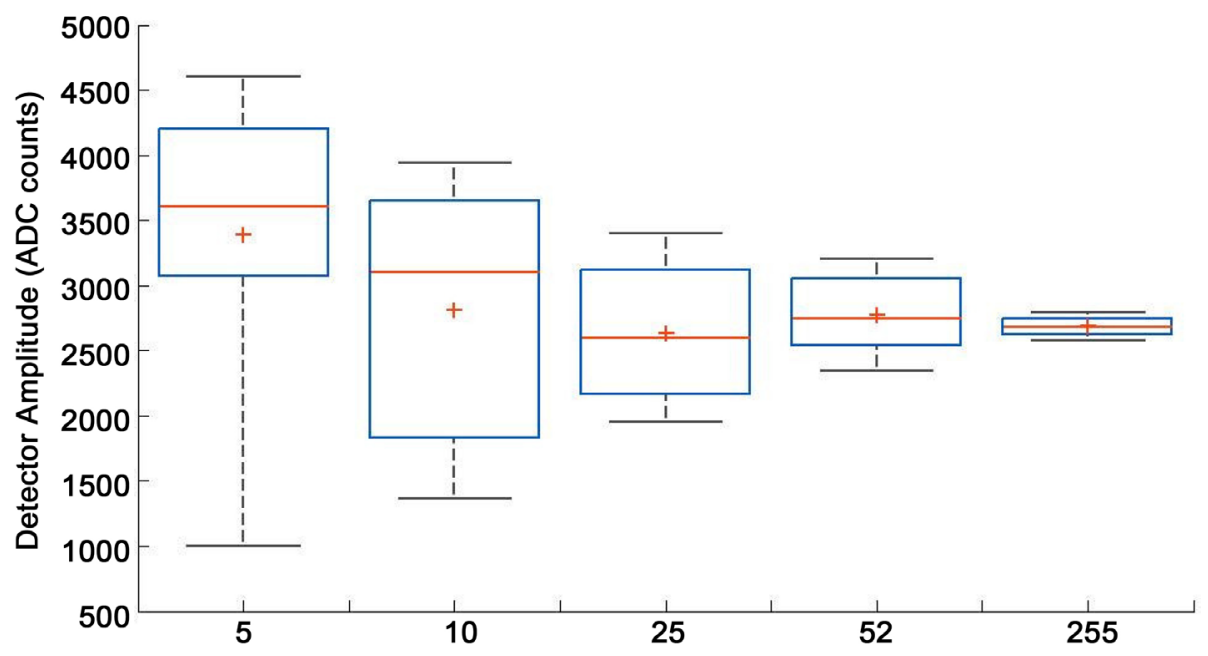

Figure 3. The box and whisker spreads of output amplitudes after dark current compensation for different number of equidistant steps. 
Figure 5 shows, as example, a simulation of spectrum of the $\mathrm{x}$-ray tube source with tungsten target, with $70 \mathrm{kVp}$ anode voltage, and built-in $2.5 \mathrm{~mm}$ plus additional $20 \mathrm{~mm}$ aluminum filter. The spectrum generated with Monte-Carlo simulation program XMIM-SIM [12] [13]. The detector current response for such difference in intensity and $\mathrm{x}$-ray energy is easier to describe as a polynomial expression.

To find the $k_{i}$ coefficients we use the data of the ramp object exposure. The data of a raw image for sensitivity compensation is $B$, the ramp test-object is represented as a matrix $R$ where each row is an array's data on the same ramp thickness. The vector $Z$ is the values proportional to $d^{i}$ from (7). Number of rows in $R$ and $Z$ is the same.

$$
B_{m, n}=\left(\begin{array}{cccc}
b_{1,1} & b_{1,2} & \cdots & b_{1, n} \\
b_{2,1} & b_{2,2} & \cdots & b_{2, n} \\
\vdots & \vdots & & \vdots \\
b_{m, 1} & b_{m, 2} & \cdots & b_{m, n}
\end{array}\right), R_{q, n}=\left(\begin{array}{cccc}
r_{1,1} & r_{1,2} & \cdots & r_{1, n} \\
r_{2,1} & r_{2,2} & \cdots & r_{2, n} \\
\vdots & \vdots & & \vdots \\
r_{q, 1} & r_{q, 2} & \cdots & r_{q, n}
\end{array}\right), Z=\left(\begin{array}{c}
z_{1} \\
z_{2} \\
\vdots \\
z_{q}
\end{array}\right), K=\left(\begin{array}{c}
k_{0} \\
k_{1} \\
k_{2} \\
k_{3}
\end{array}\right)
$$

In order to compensate the amplitude difference between all sensitive elements, we calculate an adjustment value for each pixel in $B$.

To compensate sensitivity of all elements to the same level we use a ramp as a reference

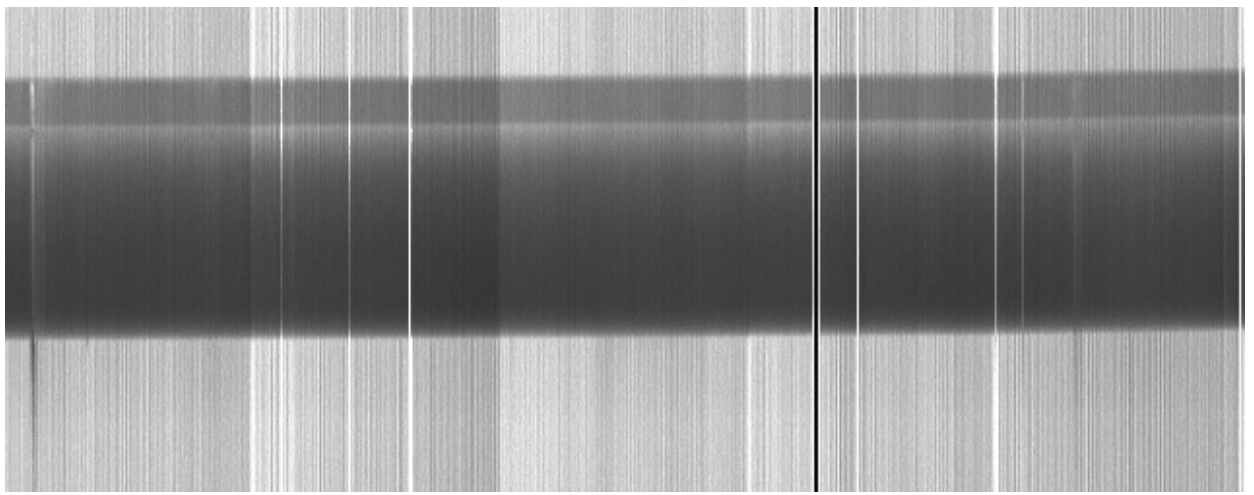

Figure 4. Reference ramp object for sensitivity adjustment of detector linear array.

X-Ray tube spectrum with tungsten target High voltage-70 kVp, current- $25 \mathrm{~mA}$, solid angle- $0.05 \mathrm{sr}$.

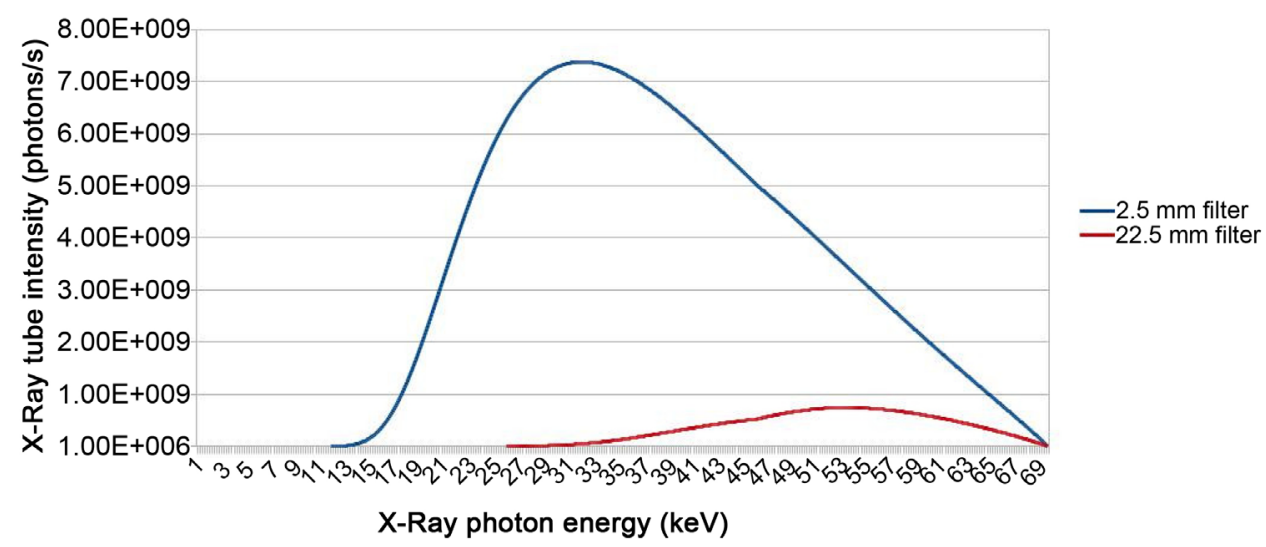

Figure 5. Simulation of an x-ray tube spectrum with different aluminum filters. 
and for each raw image pixel we calculate a minimum index of the absolute difference between pixel and ramp matrix column. Column vector $Y$ is formed for each element of matrix $B$.

$$
\begin{aligned}
& \forall b_{i, j}: \bar{Y}_{p}=\left(\begin{array}{c}
\left|b_{i, j}-r_{1, j}\right| \\
\left|b_{i, j}-r_{2, j}\right| \\
\vdots \\
\left|b_{i, j}-r_{q, j}\right|
\end{array}\right),(p=1,2, \cdots, q) \\
& \forall p=1,2, \cdots, q \exists f: f=\arg \min _{p} \bar{Y}_{p}
\end{aligned}
$$

As a result the compensated pixel $b^{\prime}$ will be

$$
b_{i, j}^{\prime}=\frac{b_{i, j} \times z_{i}}{r_{f, j}}
$$

In order to increase a number of rows in matrix $R$ the interpolation could be done by calculating additional data for all rows. Equations (11) and (7) allow to find $k_{i}$ coefficients with linear least square approach and recalculate row data for expanded $Z$.

$$
\left(\begin{array}{l}
k_{3} \\
k_{2} \\
k_{1} \\
k_{0}
\end{array}\right)=\left(\begin{array}{cccc}
z_{1}^{3} & z_{1}^{2} & z_{1} & 1 \\
z_{2}^{3} & z_{2}^{2} & z_{2} & 1 \\
\vdots & \vdots & \vdots & \vdots \\
z_{q}^{3} & z_{q}^{2} & z_{q} & 1
\end{array}\right)^{-1} \times\left(\begin{array}{c}
r_{1,1} \\
r_{2,1} \\
\vdots \\
r_{q, 1}
\end{array}\right)
$$

The resulted image formed from pixels, calculated from equation 10 is represented as "for processing" in DICOM 3.0 format for further PACS systems.

\section{Examples of Sensitivity Adjustment on Raw Images}

Images described below were obtained with 3 modules which have minimum pixel intensity fluctuation due to scanner array movement and defects in input channels of ASIC which affects on output signals. Raw image example with compensated dark currents is showed in Figure 6 together with adjusted sensitivity by using the ramp object calibration and by MIRE [14] algorithm. The scan was done from top to the bottom and the exposure time was 1.4 seconds. Algorithms which use only image data itself without additional information will leave artifacts in regions with significant intensity gradients.

The acquisition parameters were:

- clock frequency: $19 \mathrm{MHz}$ (1.2 MHz per detector module)

- raw scan-line formation time: 444 us

- scan-line formation time after resample to $1: 1$ scale: $2.2 \mathrm{~ms}$

- detector voltage: $-20 \mathrm{~V}$

- detector operation temperature: $+12.5^{\circ} \mathrm{C}$.

- high voltage of $\mathrm{x}$-ray tube: $80 \mathrm{kVp}$.

- focal distance: $100 \mathrm{~cm}$.

One of the main characteristics of the $\mathrm{x}$-ray imaging system is a modular transfer 

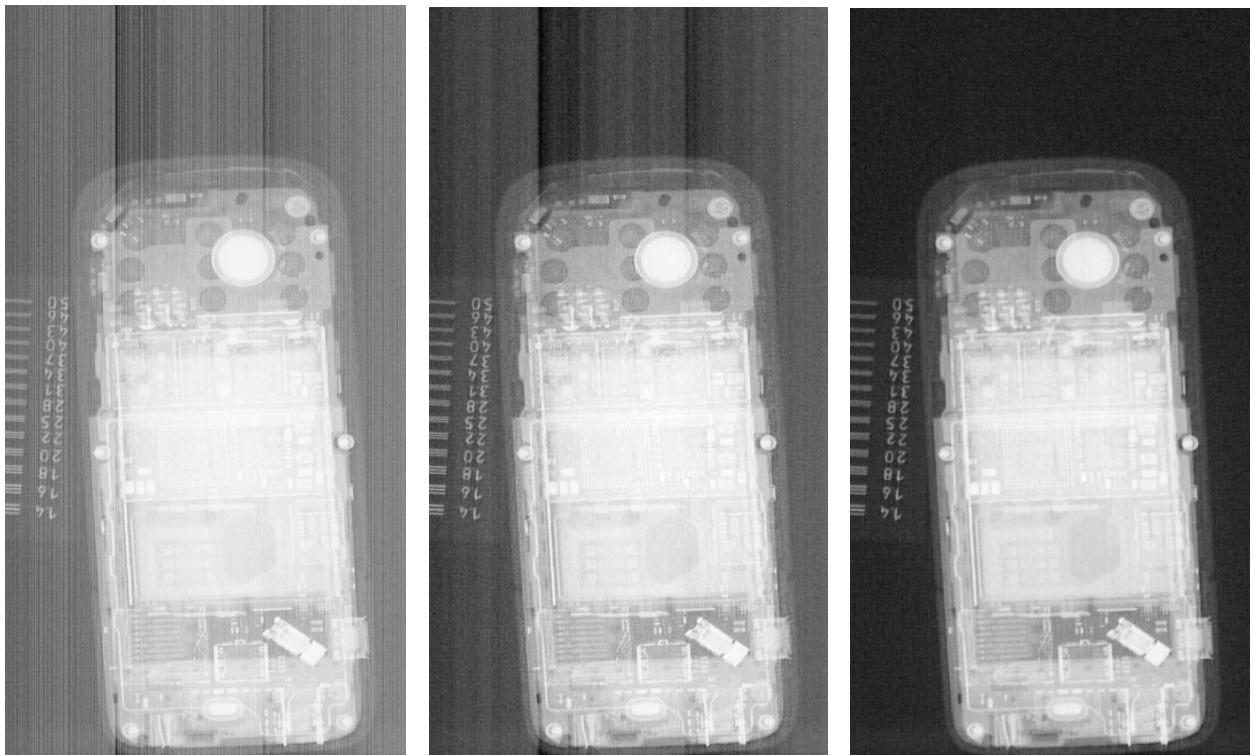

Figure 6. Example of sensitivity adjustment, from left to right: raw image, adjusted with MIRE algorithm, adjusted using ramp object.

function (MTF), which is measured by means of slanted edge test object throughout the additional $20 \mathrm{~mm}$ aluminium filter. High voltage of x-ray tube during MTF measurement was $70 \mathrm{kVp}$ and the distance between $\mathrm{x}$-ray tube and test-object was $95 \mathrm{~cm}$. Figure 7 represents raw and adjusted images of slanted sharp-edge object oriented in parallel and perpendicular to array detector during the scan. Resulted MTF functions were calculated in ImageJ [15] program using two plugins SE_MTF [16] and COQ [17] [18].

Examples of images before and after sensitivity adjustment are shown on Figure 8 from full length of linear array of 16 sensitive modules and 2048 sensitive elements. Resulted MTF is represented in Figure 9 for two rescale filters (Cubic and Lanczos) of raw image obtained with the same exposition parameters.

The $10 \%$ level of MTF function lies within the $2-3 \mathrm{lp} / \mathrm{mm}$ range for both vertical and horizontal orientation of the slanted edge in agreement with the spatial resolution pattern image.

Another very important evaluation parameter on development stage of a scanning array is a normalized noise power spectrum (NNPS). It is fluctuating because of different characteristics of detector modules and ASICs. NNPS is presented in Figure 10 for the resulted image with $20 \mathrm{~mm}$ aluminum filter under uniform exposition for vertical, horizontal and radial direction of the scan. NPS data were measured with 3 modules (384 sensitive elements). The peak in vertical direction represents periodic electronic noise in output signals from some ASICs.

The presented technique of sensitivity adjustment has several disadvantages such as need a calibration ramp or other type of uniform object. The misalignment between a ramp object and a linear array may cause additional artifacts in a final image after the adjustment procedure. In case of the large thickness of the object the brightest or darkest 

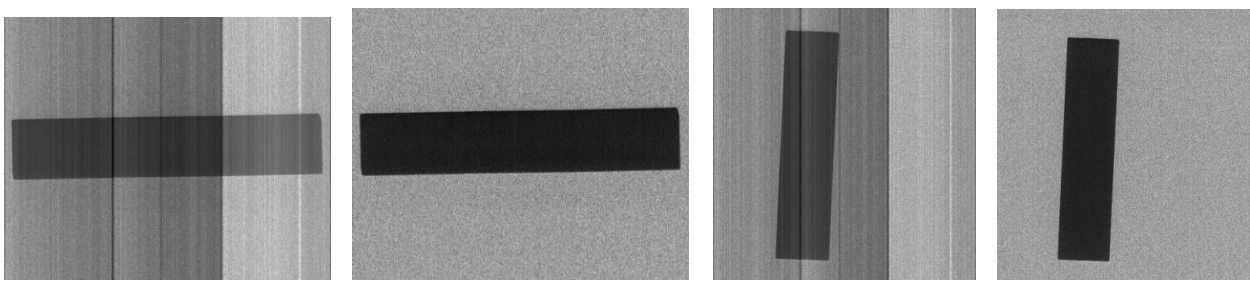

Figure 7. Images of slanted edge object before and after detector array sensitivity adjustment.
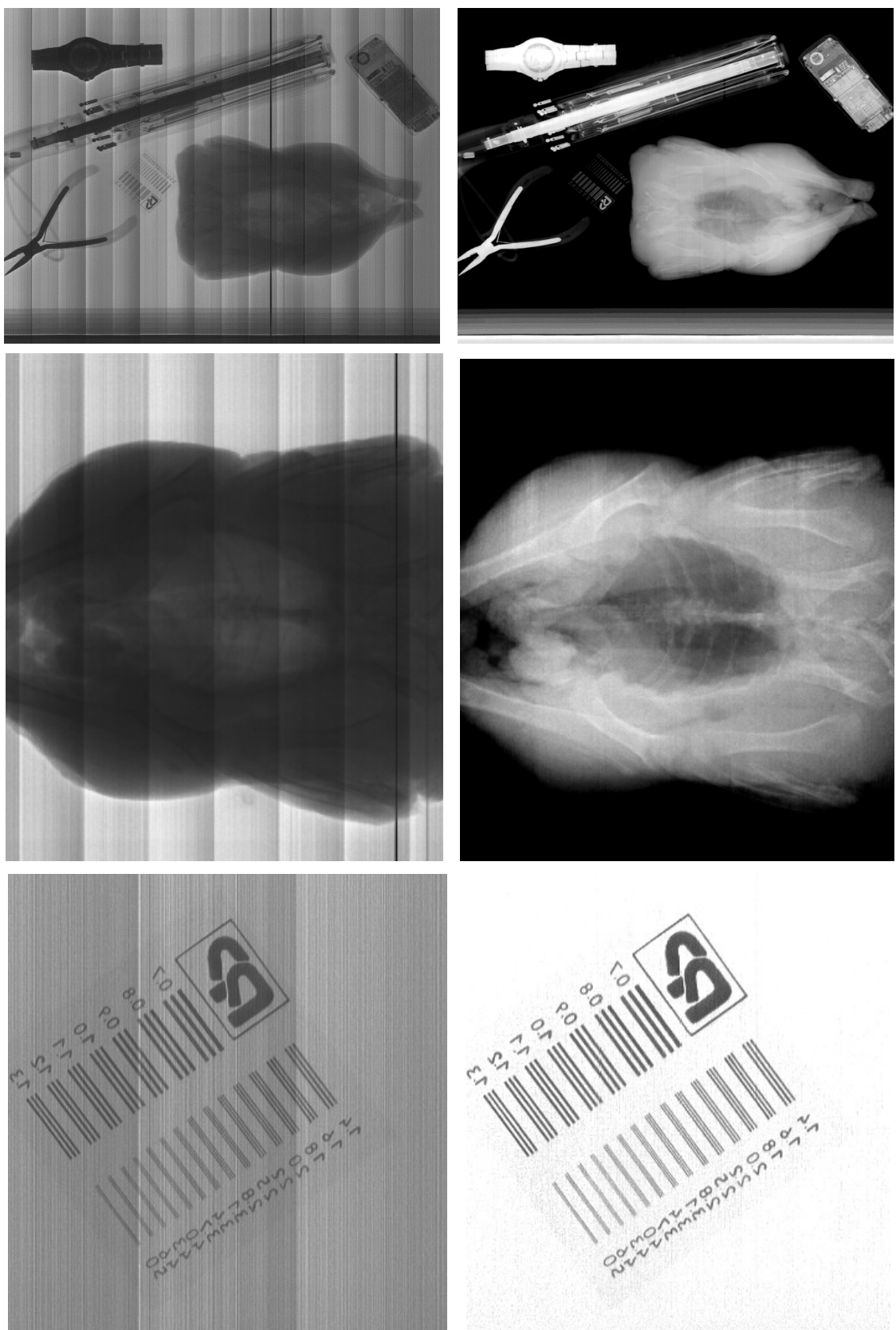

Figure 8. Examples of images before and after sensitivity adjustment.

pixels in the image could be adjusted wrongly and some artifacts will still remain. 


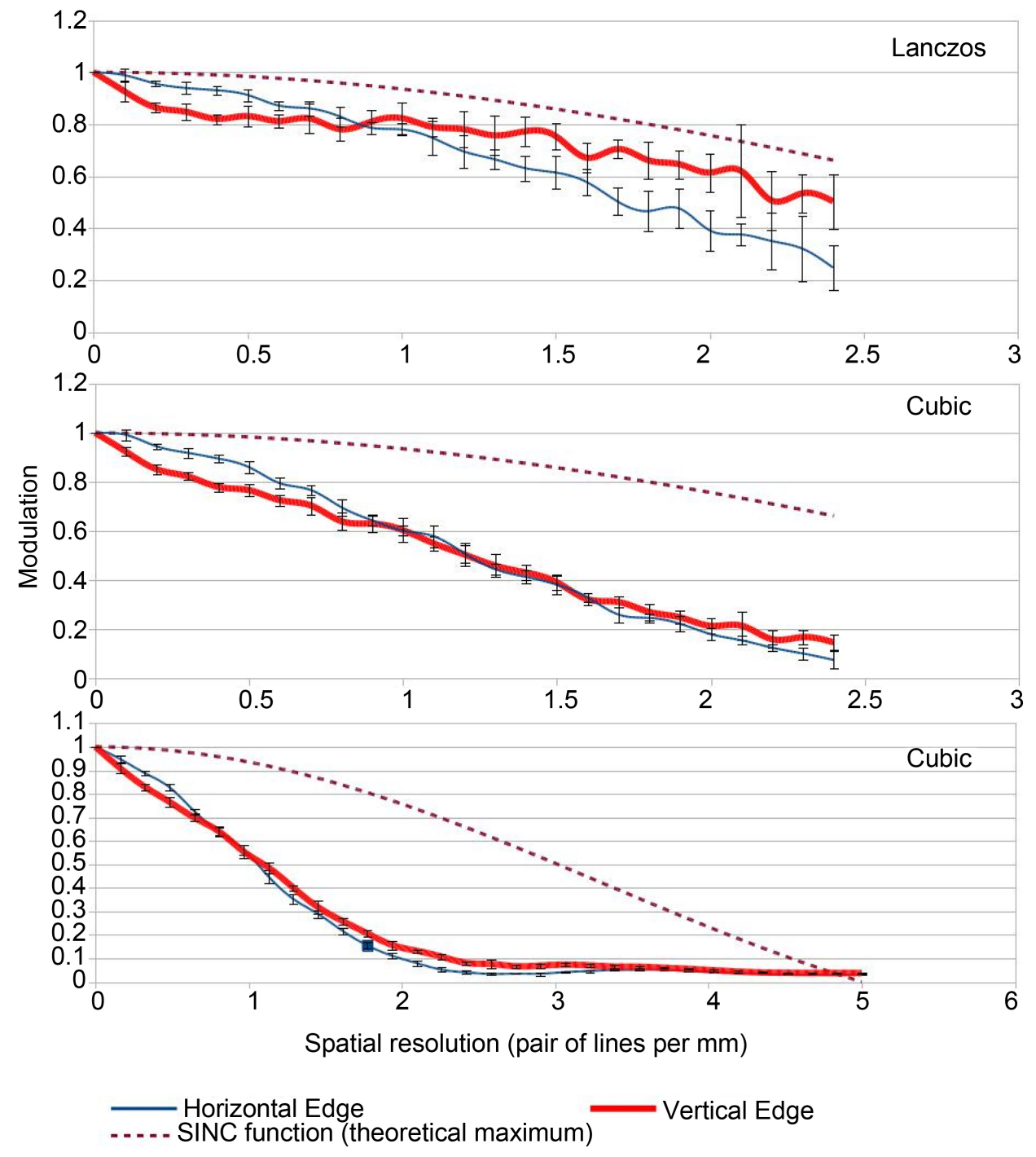

Figure 9. Modular transfer function for two convolution matrices Cubic and Lanczos (raw image rescale filters) with the same exposure and acquisition parameters.

\section{Results}

There are two algorithms described in article: dark current compensation algorithm to reduce signal variance on each detective module, and sensitivity adjustment to compensate sensitivity of each detection element. The represented algorithm of dark current compensation allows minimizing spread of output 12-bit ADC values up to 30 ADC counts (sigma of Gaussian distribution). The noise reduction of the raw image data by sensitivity adjustment via reference ramp object could be a key element of a program algorithm when it's impossible to use data of the raw image itself and the resulted image quality is crucial. Different exposure parameters, such as different high voltages of x-ray tube and different anode currents can be adjusted well with the usage of several ramp objects. This procedure must be adopted initially before any other exposures with real objects or patients. The same technique could be used with number of 


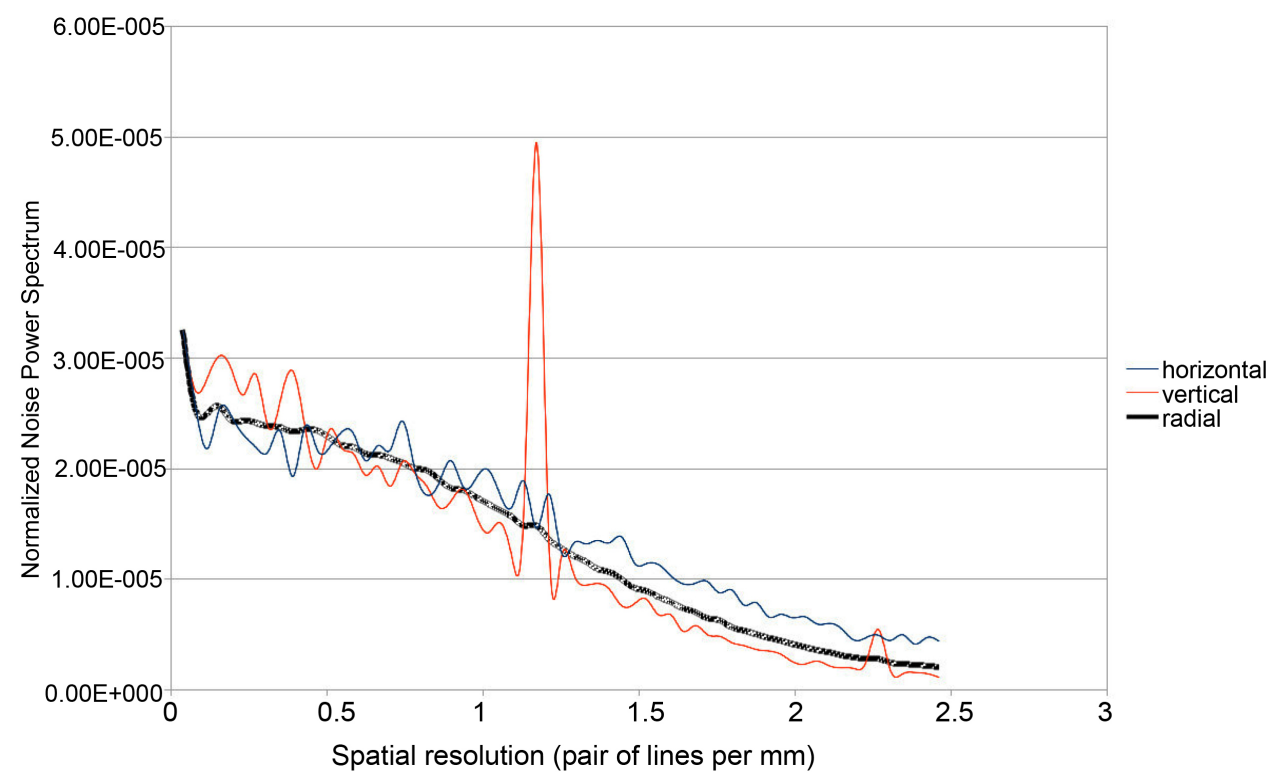

Figure 10. Normalized noise power spectrum for horizontal, vertical and radial directions of the scan.

images with uniform exposure of the whole region of interest. The modular transfer function and spatial resolution for GaAs linear array detector were measured with dark current compensation and sensitivity adjustment of detector elements using the developed algorithms with reasonable output. For 200 um pitch sensitive array element 10\% MTF has value between 2 - 3 pair of lines per $\mathrm{mm}$.

\section{References}

[1] Ardashev, E.N., Gorokhov, S.A., Polkovnikov, M.K., Lobanov, I.S. and Vorobiev, A.P. (2011) A Portable X-Ray Apparatus with GaAs Linear Array. Nuclear Instruments and Methods in Physics Research Section A, 648, S8-S11. http://dx.doi.org/10.1016/j.nima.2011.02.030

[2] Dubecký, F., Perd'ochová, A., Ščepko, P., et al. (2005) Digital X-Ray Portable Scanner Based on Monolithic Semi-Insulating GaAs Detectors: General Description and First "Quantum" Images. Nuclear Instruments and Methods in Physics Research Section A, 546, 118-124. http://dx.doi.org/10.1016/j.nima.2005.03.020

[3] Dvoryankin, V.F., Dikaev, Yu.M., Krikunov, A.I., Panova, T.M. and Telegin, A.A. (2004) Multielement X-Ray Row Detector on GaAs with Spatial Resolution of $108 \mu \mathrm{m}$. Nuclear Instruments and Methods in Physics Research Section A, 531, 87-88. http://dx.doi.org/10.1016/j.nima.2004.05.097

[4] Dvoryankin, V.F., Dvoryankina, G.G., Dikaev, Yu.M., Ermakov, M.G., Kudryashov, A.A., Petrov, A.G. and Telegin, A.A. (2012). Photovoltaic GaAs Detectors for Digital X-Ray Imaging, Mammography - Recent Advances, Dr. Nachiko Uchiyama (Ed.), InTech. http://dx.doi.org/10.5772/32431

[5] Tendero, Y. and Gilles, J. (2012) ADMIRE: A Locally Adaptive Single-Image, Non-Uniformity Correction and Denoising Algorithm: Application to Uncooled IR Camera. Proceedings SPIE 8353, Infrared Technology and Applications XXXVIII, 835310.

[6] Harris, J.G. and Chiang, Y.M. (1999) Nonuniformity Correction of Infrared Image Se- 
quences Using the Constant-Statistics Constraint. IEEE Transactions on Image Processing, 8, 1148-1151. http://dx.doi.org/10.1109/83.777098

[7] Li, H.-C. and Song, H.-F. (2011) A Relative Radiometric Correction Method for Linear Array Push-Broom Imagery. Proceedings SPIE 8194, International Symposium on Photoelectronic Detection and Imaging 2011: Advances in Imaging Detectors and Applications, 81941L. http://dx.doi.org/10.1117/12.900195

[8] Ma, W.H., Dong, T., Tian, H. and Ni, J.P. (2014) Line-Scan CCD Camera Calibration in 2D Coordinate Measurement. Optik - International Journal for Light and Electron Optics, 125, 4795-4798. http://dx.doi.org/10.1016/j.ijleo.2014.04.057

[9] Tyazhev, A.V., Budnitsky, D.L., Koretskay, O.B., Novikov, V.A., Okaevich, L.S., Potapov, A.I., Tolbanov, O.P. and Vorobiev, A.P. (2003) GaAs Radiation Imaging Detectors with an Active Layer Thickness up to $1 \mathrm{~mm}$. Nuclear Instruments and Methods in Physics Research Section A, 509, 34-39. http://dx.doi.org/10.1016/S0168-9002(03)01545-6

[10] Veale, M.C., Bell, S.J., Duarte, D.D., French, M.J., Schneider, A., Seller, P., Wilson, M.D., Lozinskaya, A.D., Novikov, V.A., Tolbanov, O.P., Tyazhev, A. and Zarubin, A.N. (2014) Chromium Compensated Gallium Arsenide Detectors for X-Ray and $\gamma$-Ray Spectroscopic Imaging. Nuclear Instruments and Methods in Physics Research Section A, 762, 6-14. http://dx.doi.org/10.1016/j.nima.2014.03.033

[11] Borodin, D.V., Osipov, V.V., Shushkevich, N.A. and Lopuhin A.A. (2003) Testing the Readout Integrated Circuits. Applied Physics, 1, 154-156 (in Russian). http://applphys.orion-ir.ru

[12] XMI-MSIM: Monte Carlo Simulation of Energy-Dispersive X-Ray Fluorescence Spectrometers. http://github.com/tschoonj/xmimsim

[13] Schoonjans, T., Brunetti, A., Vincze, L., et al. (2011) The Xraylib Library for X-Ray-Matter Interactions. Recent Developments. Spectrochimica Acta B, 66, 776-784. http://dx.doi.org/10.1016/j.sab.2011.09.011

[14] Tendero, Y., Landeau, S. and Gilles, J. (2012) Non-Uniformity Correction of Infrared Images by Midway Equalization. Image Processing On Line, 2, 134-146. http://dx.doi.org/10.5201/ipol.2012.glmt-mire

[15] ImageJ. https://imagej.nih.gov/ij/index.html

[16] SE_MTFplugin. http://rsb.info.nih.gov/ij/plugins/se-mtf/index.html

[17] Nitrosi, A., Bertolini, M., et al. (2009) Application of QC_DR Software for Acceptance Testing and Routine Quality Control of Direct Digital Radiography Systems: Initial Experiences using the Italian Association of Physicist in Medicine Quality Control Protocol. Journal of Digital Imaging, 22, 656-666. http://dx.doi.org/10.1007/s10278-008-9150-z

[18] Donini, B., Rivetti, S., Lanconelli, N., et al. (2014) Free Software for Performing Physical Analysis of Systems for Digital Radiography and Mammography. Medical Physics, 41, Article ID: 051903. http://dx.doi.org/10.1118/1.4870955 
Submit or recommend next manuscript to SCIRP and we will provide best service for you:

Accepting pre-submission inquiries through Email, Facebook, LinkedIn, Twitter, etc. A wide selection of journals (inclusive of 9 subjects, more than 200 journals)

Providing 24-hour high-quality service

User-friendly online submission system

Fair and swift peer-review system

Efficient typesetting and proofreading procedure

Display of the result of downloads and visits, as well as the number of cited articles

Maximum dissemination of your research work

Submit your manuscript at: http://papersubmission.scirp.org/

Or contact jbise@scirp.org 